\title{
Larva migrans visceral como causa de síndrome febril prolongado y eosinofilia severa. Reporte de un caso
}

\author{
Visceral larva migrans as a cause of prolonged febrile \\ syndrome and severe eosinophilia. A case report
}

\author{
Luis Alberto Chamorro Noceda ${ }^{1}$ \\ ${ }^{1}$ Instituto de Previsión Social, Hospital Central, Servicio de Pediatría. Asunción, Paraguay.
}

\section{RESUMEN}

Toxocara canis y catis son helmintos que parasitan el intestino del perro o del gato, sus huéspedes definitivos. Las hembras eliminan huevos infectantes al medio ambiente, que, ingeridos accidentalmente por el ser humano, sólo desarrollan el estado larvario en etapa embrionaria y causan la toxocariasis. Esta es una zoonosis extendida por todo el mundo, especialmente en aquellas áreas donde prevalecen condiciones deficientes de hábitat e higiene. El cuadro clínico puede variar desde formas asintomáticas a la larva migrans visceral (LMV) o larva migrans ocular (LMO), debidas a la diseminación de larvas embrionadas en el hígado, pulmones y en menor medida el SNC. La LMV se caracteriza clínicamente por la presencia de hepatomegalia, infiltración pulmonar con tos, eosinofilia de variada intensidad, hiper Ig E y anticuerpos Ig G anti-toxocara. Por no ser la fiebre un síntoma clínico destacado, se presenta el siguiente caso clínico.

Palabras claves: Toxocariasis, larva migrans visceral, síndrome febril prolongado

\section{INTRODUCCIÓN}

Los toxocara canis y catis son helmintos del tipo áscaris, que parasitan el intestino delgado del perro y del gato respectivamente, que son sus huéspedes definitivos. Las hembras eliminan huevos en forma diaria, que en el suelo del ambiente tardan 3-4 semanas en desarrollarse al estado larvario

\section{ABSTRACT}

Toxocara canis and catis are helminths that parasitize the intestine of dogs or cats, their definitive hosts. Females shed infective eggs into the environment, which, when accidentally ingested by humans, only develop the embryonic larval stage and cause toxocariasis. This is a zoonosis that is widespread throughout the world, especially in those areas where poor habitat and hygienic conditions prevail. The clinical picture can vary from asymptomatic forms to visceral larva migrans (VLM) or ocular larva migrans (OLM), due to the dissemination of embryonic larvae in the liver, lungs and to a lesser extent the CNS. VLM is clinically characterized by the presence of hepatomegaly, pulmonary infiltration with cough, eosinophilia of varying intensity, hyper Ig E and Ig G antitoxocara antibodies. Since fever is not a prominent clinical symptom, the following clinical case is presented.

Key words: Toxocariasis, visceral larva migrans, prolonged febrile syndrome.

infectante. Éstas, al ser ingeridas por una variedad de especies animales como el ser humano, nunca desarrollarán en su organismo el estadio adulto definitivo, por ser huéspedes intermediarios, también llamados huéspedes paratenicos ${ }^{(1)}$. El primer reporte sobre toxocaríasis fue en 1.952 por

Correspondencia: Luis Alberto Chamorro Noceda Correo: lachamorro18@gmail.com Recibido: 06/10/2020 Aceptado:24/06/2021 DOI: https://doi.org/10.31698/ped.48022021010

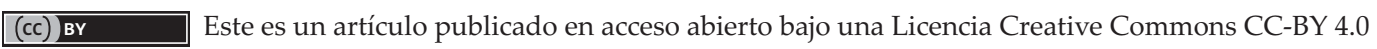


Beaver, en tres niños que presentaban alta eosinofilia y lesiones granulomatosas crónicas en el hígado e identificaron que las larvas encontradas en el órgano eran de toxocara canis, por lo que designaron a la novel enfermedad como "larva migrans visceral". El ser humano se infecta al ingerir de forma accidental los huevos infectantes de toxocara, que contaminan frutas y verduras mal lavadas, carne o hígado no bien cocidos; en especial los niños, que tienen el hábito de practicar la onicofagia o la geofagia, y quienes viven en un ambiente contaminado debido a condiciones de pobreza socioeconómica ${ }^{(2)}$. Siendo una zoonosis extendida a nivel mundial, constituye una amenaza silenciosa, con gran impacto en la salud pública ${ }^{(3)}$. Se estima que millones de personas en Estados Unidos han sido expuestas al parásito toxocara, pero se desconoce cuántos de ellos se enfermaron luego de la infección ${ }^{(4)}$. La seroprevalencia de la infección oscila de $19 \%$ a $86 \%$, según estudios realizados en diferentes países, siendo más afectados los niños que conviven con cachorros en su hogar y en estrecho contacto con el suelo contaminado; o que viven en condiciones sociales desfavorables ${ }^{(5,6)}$. La infección larvaria por toxocara es asintomática en la mayoría de los casos, o puede manifestarse como enfermedad, dando diversos cuadros clínicos como la larva migrans visceral (LMV), la larva migrans ocular $(\mathrm{LMO})^{(7)}$ o menos frecuentemente afectando al SNC. La LMV se manifiesta con síntomas y signos diversos que dificultan y atrasan el diagnóstico, siendo el mismo necesario para un tratamiento adecuado. Los signos y síntomas clínicos principales son: afectación del estado general, pérdida de peso, palidez, hepatomegalia, en ocasiones adenomegalias y algunas manifestaciones atópicas como sibilancias, rash cutáneo, alergia alimentaria o artralgia, dolor abdominal recurrente, tos y fiebre; y en el hemograma la presencia de anemia ferropénica, eosinofilia e inmunoglobulinas específicas Ig G4 e Ig $\mathrm{E}$ anti-toxocaras ${ }^{(8)}$. La fiebre, por ser un síntoma poco destacable de la LMV, está escasamente considerada en la literatura, lo que motiva la presentación del presente caso clínico. Teniendo nuestro país una gran parte de su población viviendo en condiciones de pobreza, sin el saneamiento ambiental adecuado, contando muchos hogares con la presencia de perros mascotas; es necesario sospechar la posibilidad de toxocariasis si el hemograma realizado a un niño revela eosinofilia, aún en ausencia de síntomas, especialmente si ella es elevada, para hacer el estudio que lleve al diagnóstico correspondiente y el tratamiento adecuado.

\section{CASO CLÍNICO}

Paciente de 1 año 7 meses de edad, de sexo femenino, procedente de Capiatá, con historia de fiebre intermitente de 2 semanas de evolución. La paciente presentó varios picos febriles al día, graduados, en ocasiones hasta $39^{\circ} \mathrm{C}$; acompañada de tos seca al inicio y luego catarral. Consultó a los 2 días del inicio del cuadro con facultativo, quién al realizarle un hemograma $(29 / 09 / 18)$ y constatar leucocitosis: 20.500/mm3, N:45\%; L:30\%; M:4\%; E:20\% (eosinófilos absolutos 4.100/mm3); Hg:10.1 gr/dl y datos de ferropenia, indica tratamiento con Ceftriaxona $\mathrm{e} / \mathrm{v}$ por 3 días, Claritromicina por 10 días y Dipirona. Ante la falta de respuesta a la medicación por persistencia de la fiebre, acude nuevamente al facultativo quién le realiza otro hemograma (09/10/18) con el siguiente resultado: $\mathrm{Hg}: 9,8 \mathrm{~g} / \mathrm{dl}$, GR: $3.790 .000 / \mathrm{mm} 3$, plaquetas: $556.000 / \mathrm{mm} 3$; G.B:59.200/mm3, N:2\%；L:4\%; M:2\%; E:92\% (eosinófilos absolutos 54.280), por lo cual lo remite al HCIPS, ingresando a Sala de Lactantes el 10/10/18 con TA: 37‥8 C; peso: $9 \mathrm{~kg}(\mathrm{P} 3)$; talla $82 \mathrm{~cm}(\mathrm{P} 50)$; CC:47 $\mathrm{cm}(\mathrm{P} 50)$. Al examen físico llama la atención palidez cutáneo-mucosa y distención abdominal de difícil palpación, por la escasa colaboración de la paciente. En los antecedentes familiares no se obtuvieron datos de valor y como antecedentes personales dos internaciones previas por gastroenteritis. Condiciones de hábitat: vive en casa de material, con agua potable, no tiene mascotas en la casa, pero refiere contacto con perros de familiares. Ingresa con los diagnósticos de Síndrome febril prolongado, anemia y eosinofilia severa de etiología a determinar. Los análisis realizados el 10/10/18 revelaron: $\mathrm{Hg}: 8.4$ g/dl; GR:3.500.000/mm3; GB:70.510/mm3; NS:12\%; L:10\%; M:3\%; E:75\% (eosinófilos absolutos 52.882) y hematimetría con datos de ferropenia; plaquetas: 580.000/mm3; GOT:147 UI/L; GPT:245 UI/L; creatinina, urea, electrolitos, orina, crasis sanguínea, pruebas de STORCH: todas con valores normales, y estudio coproparasitario negativo para vermes y protozoarios. El examen físico del día siguiente reveló hepatomegalia a $8 \mathrm{~cm}$. del reborde costal y punta de bazo. Ante esos datos se reinterroga a los 
padres, quienes reconocen que la niña tenía el hábito de la geofagia. Con esos antecedentes y la elevada eosinofilia, se sospechó clínicamente la posibilidad de larva migrans visceral (toxocaríasis) por lo cual se inició tratamiento con Albendazol $15 \mathrm{mg} / \mathrm{kg} / \mathrm{día}$, al tercer día del ingreso; solicitándose IgE, cuyo valor fue mayor a $2.000 \mathrm{UI} / \mathrm{ml}$, e IgG anti-toxocaras, por la técnica de inmunoabsorciòn de ELISA, que fue positiva. La ecografía abdominal reveló: Hepatomegalia con formaciones micronodulares distribuidas en gran parte del hígado, adenopatías de diverso tamaño en el hilio hepático, esplenomegalia leve con formaciones micronodulares. La paciente continuó con picos de fiebre de 38 a $39^{\circ} \mathrm{C}$, cediendo la misma al cuarto día de internación y al segundo día de iniciada la medicación con Albendazol. Nuevos estudios laboratoriales: GPT: $181 \mathrm{UI} / \mathrm{ml}$; GOT: 120 $\mathrm{UI} / \mathrm{ml}$; Proteinograma: proteínas totales $8.1 \mathrm{~g} / \mathrm{dl}$; albúmina: $3.8 \mathrm{~g} / \mathrm{dl}$; globulinas: $4.3 \mathrm{~g} / \mathrm{dl}$, relación A/G 0.9; el proteinograma electroforético reveló hipergammaglobulinemia: proteína total $8.20 \mathrm{~g} / \mathrm{dl}$ alb. $3.15 \mathrm{~g} / \mathrm{dl}$ (3.70-4.83); gamaglobulina $2.48 \mathrm{~g} / \mathrm{dl}$ (0.84-1.36). La Radiografía de tórax demostró discreta infiltración intersticial y el estudio de fondo de ojo fue normal. Fue dada de alta el 18/10/19, al $8^{\circ}$ día de internación, con un peso de 9.450 grs. con los diagnósticos de: Larva migrans visceral por toxocaríasis, anemia microcítica hipocrómica moderada y desnutrición calórico-proteica moderada; con indicación de ferroterapia con sulfato ferroso a $3 \mathrm{mg} / \mathrm{kg} /$ día, polivitamínico y de repetir el tratamiento con Albendazol 10 días más. El hemograma realizado al alta reveló GR: 3.470.000/mm3; Hg: $8.2 \mathrm{~g} / \mathrm{dl}$, hematimetría con datos de ferropenia; G.B: 65.790/mm3; NS: 4\%; L: 10\%; M: 2\%; E: 84\% (eosinófilos absolutos 55.263/mm3). En el control del 02/11/18 el hígado se había reducido de tamaño a $5 \mathrm{~cm}$ del RCD y el hemograma indicó Hg: $9.3 \mathrm{~g} / \mathrm{dl}$; G.R: 3.860.000/mm3; G.B: 36.770/mm3; NS:20\%; L:18\%; M:4\%; E: 58\% (eosinófilos absolutos 21.323). En la ecografía abdominal persistía la hepatomegalia con imágenes micronodulares, por lo cual se indicó nuevo plan de tratamiento con Albendazol por 5 días más; y en su último control del 15/11/18 el hemograma reveló: Hg: 9.9 g/dl; G.R: 3.920.000/mm3; G.B: 23.000/ mm3; NS:25\%; L:32\%; M:4\%; E:39\% (eosinófilos absolutos 8.970/mm3). Hepatomegalia a $3.5 \mathrm{~cm}$ del RCD y a la ecografía reducción del número de imágenes micronodulares.
La paciente no acudió a controles posteriores programados.

\section{DISCUSIÓN}

El toxocara canis y el toxocara catis son helmintos áscaris, midiendo los adultos machos de 4 a $10 \mathrm{cms} \mathrm{y}$ las hembras 6.5 a $18 \mathrm{cms}$. de longitud. Parasitan el intestino delgado de perros y gatos respectivamente, que son sus huéspedes definitivos, desde donde las hembras eliminan con las heces más de 200.000 huevos al día, los cuales son esparcidos al suelo del ambiente, sin ser aún infectivos. En este medio tardan 3-6 semanas en llegar al estado embrionado. En este momento adquieren el potencial riesgo de infectar, si son ingeridos accidentalmente por una variedad de huéspedes intermediarios o paratenicos, como aves caseras, ratones, ratas, cerdos, primates y los seres humanos ${ }^{(9)}$. El suelo contaminado no es la única fuente de infección; también ocurre por la ingestión de hígado de huéspedes paratenicos parasitados o de verduras contaminadas por aguas de regadío no tratadas. Una vez ingeridos los huevos embrionados eclosionan en el duodeno del huésped paratenico, liberando las larvas que no llegarán al estado adulto al no completar su ciclo de maduración. Estas penetran la mucosa del duodeno e inician el ciclo de la migración larvaria, llegando al hígado a través de la circulación portal y por el sistema venoso de la cava inferior a los pulmones. Los órganos más afectados son el hígado, los pulmones, los ojos, los ganglios, el SNC y el corazón. $\mathrm{Al}$ ser detenidas las larvas en esos tejidos se rodean de un granuloma formado por eosinófilos, macrófagos, histiocitos y fibras colágenas. La infección con el parásito produce la activación de la respuesta inmunitaria de tipo mixta, donde el tipo TH1 da una hipersensibilidad retardada, que justifica la formación de granulomas hepáticos; una respuesta polarizada de $\mathrm{TH} 2 \mathrm{CD} 4+$, con la producción de las citosinas L5 y GM-CSF, elevando la producción de eosinófilos y de la L4 que estimula a los linfocitos B, promoviendo el aumento de IgE específica, mediada por moléculas HLA clase II. Las células fagocíticas abundan y las larvas atrapadas en los granulomas eosinofílicos pueden degenerar y calcificarse ${ }^{(10)}$, ocurriendo a veces formación de abscesos hepáticos secundarios en los granulomas, más frecuentemente en el lóbulo derecho, 
generalmente causados por estafilococos áureos ${ }^{(11)}$. La larva libera diariamente a la sangre aproximadamente $2 \mathrm{ng}$ /día de glicoproteínas antigénicas excretoras-secretoras, que generan anticuerpos específicos de tipo IgG4 e IgE y otras glicoproteínas antigénicas de tipo alergénicas, que son responsables de las reacciones atópicas que presentan muchos pacientes ${ }^{(12)}$. La toxocariasis es una zoonosis extendida mundialmente, afectando al ser humano a diferentes edades, principalmente a los niños, en su mayoría pobres, de países del área tropical o subtropical ${ }^{(13)} \mathrm{y}$ de las áreas rurales de países industrializados ${ }^{(14)}$. En una revisión sistemática de 250 estudios de 71 países, desde 1980 hasta 2019, mediante metaanálisis, la tasa global de toxocaríasis fue de $19 \%$, siendo la prevalencia más alta en África con $37.7 \%$ y la más baja en el mediterráneo oriental con $8.2 \%$. La seroprevalencia combinada para otras regiones de la OMS fue de $34.1 \%$ en el sudeste asiático; $24.2 \%$ en el Pacífico Occidental; $22.8 \%$ en estadounidenses y $10.5 \%$ en regiones europeas ${ }^{(15)}$. El ser humano adulto y en particular los niños de 1 a 4 años están más expuestos a contraer la infección al exponerse al suelo de un medio ambiente muy contaminado y en particular si conviven con perros, especialmente cachorros, que se parasitan por vía transplacentaria en el $98 \%$ de los casos; siendo capaces a las 3 semanas del nacimiento de albergar en su intestino delgado vermes adultos y por tanto de eliminar huevos infectantes ${ }^{(16)}$; también cuando hay pocas normas de higiene personal, constituyendo la toxocaríasis una amenaza silenciosa con un impacto progresivo en la salud pública ${ }^{(1)}$. La toxocariasis, en su mayor parte, es una infección silente, asintomática, benigna y autolimitada, y por tanto de no fácil diagnóstico. Deja anticuerpos específicos residuales ${ }^{(12)}$, o puede manifestarse como enfermedad, adoptando la forma de larva migrans visceral (LMV), larva migrans ocular (LMO) o afectando al SNC en la forma de neurotoxocarìasis (ver figura 1) y más raramente al corazón como miocarditis, pericarditis o endocarditis de Löeffler ${ }^{(17)}$. Canese, et al., en una investigación realizada encontraron la presencia de $53 \%$ de huevos de toxocara canis en muestras de arena tomadas de las 51 plazas y parques de Asunción, concluyendo que dichos lugares se constituyen en elevado riesgo para la salud de las personas y en especial para los niños que concurren a las mismas ${ }^{(18)} \mathrm{y}$ en una investigación posterior demostraron una seroprevalencia de $78 \%$ de toxocariasis en niños de un medio rural de Piribebuy y en un área suburbana de Itauguá, siendo más frecuente la forma asintomática en $74 \%{ }^{(19)}$. La infección por toxocariasis en distintas poblaciones varía muy ampliamente con seroprevalencias del $9.5 \%$ al $86.7 \%{ }^{(20-23)}$. La sospecha de toxocariasis es orientada por una eosinofilia elevada persistente, que se confirma con el hallazgo de anticuerpos subclase Ig G4 secreción-excreción específico TES (Toxocara excretory-secretory) ${ }^{(24)}$; pero en los países subdesarrollados es muy común la existencia de infecciones por otros helmintos, las que pueden dar reacciones cruzadas del TES nativo, teniendo por tanto menor especificidad. Esto motivó el desarrollo de TES con tres antígenos recombinantes TES-26, TES-30USM y TES-120, que tienen sensibilidad y especificidad de $100 \%$ en el diagnóstico de toxocariasis $^{(25)}$. La eosinofilia puede deberse a diversas otras etiologías y ser de causa primaria o secundaria, que es la más frecuente. Se considera eosinofilia el aumento del recuento absoluto de eosinófilos (RAE) en sangre periférica mayor a $500 / \mathrm{mm} 3$ y clasificada como leve con valores de 5001500/mm3; moderada de $1.500-5.000 / \mathrm{mm} 3$ y severa mayor a 5.000/mm3; pudiendo ser transitoria, episódica o persistente ${ }^{(26)}$. Los eosinófilos tienen una toxicidad directa a través de la liberación de varias moléculas presentes en sus gránulos citoplasmáticos, como la proteína básica mayor, la peroxidasa eosinofílica, la proteína catiónica eosinofílica ${ }^{(27)}$, que activadas juegan un papel importante durante la invasión parasitaria, en especial la proteína catiónica eosinofílica (ECP), con marcada actividad proinflamatoria contra bacterias y parásitos ${ }^{(28)}$. Entre las múltiples causas de eosinofilia los parásitos helmintos son una causa importante, pero corresponde a la toxocariasis las cifras más elevadas cuando son comparadas con parásitos intestinales sean helmintos nematodes, cestodes o trematodes ${ }^{(29,30)}$. La toxocaríasis se asoció con tos crónica en $32 \%$ de los $\operatorname{casos}^{(31)}$, así también se encontró una asociación significativa entre el asma y toxocariasis ${ }^{(32)}$ y con formas graves de asma ${ }^{(33)}$, hecho que sin embargo no fue comprobado en otros estudios $^{(34,35)}$. En el Hospital Juan P. Garraham, sobre 301 pacientes con diagnóstico de toxocaríasis encontraron $31,5 \%$ con formas oculares, afectando las mismas a un solo ojo, diagnóstico que en los niños 
pequeños tiende a hacerse en forma tardía debido ya que los mismos difícilmente manifiesten una disminución de la visión, motivando la consulta el

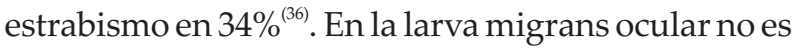
frecuente el hallazgo de anticuerpos específicos para toxocaras, siendo el diagnóstico por el estudio del fondo de ojo $^{(37)}$. Puede también afectar al SNC (neurotoxocarìasis) en forma de meningitis, meningoencefalitis o de mielitis transversa, destacándose eosinofilia tanto en sangre como en el LCR y anticuerpos Ig G para toxocara tanto en el LCR como en sangre periférica ${ }^{(38-40)}$. La eosinofilia persistente puede ser la única manifestación de la toxocariasis $^{(41,42)}$. Nuestra paciente tuvo la particularidad de haber iniciado el cuadro de LMV como un síndrome febril, acompañado al inicio de tos catarral persistente, lo que motivó el tratamiento con antibióticos como si fuese una neumonía y sin haberse tenido en cuenta la eosinofilia del $20 \%$ (RAE de $4.100 / \mathrm{mm} 3$ ) que ya presentaba en ese momento. Ante la falta de respuesta a la antibioticoterapia y debido a la persistencia de la fiebre de más de dos semanas de evolución, con empeoramiento del estado general y el descenso de la hemoglobina de $10.1 \mathrm{grs} / \mathrm{dl}$ a $9.8 \mathrm{grs} / \mathrm{dl}$, fue internada con los diagnósticos de: síndrome febril prolongado, anemia ferropénica y eosinofilia severa de causa a determinar. El hemograma al ingreso reveló severa eosinofilia de $75 \%$ y RAE de $52.882 / \mathrm{mm} 3$, hemoglobina de $8.4 \mathrm{grs} / \mathrm{dl}$. El interrogatorio dirigido, donde resaltó el antecedente de la convivencia con perro y el hábito de geofagia, llevó la sospecha de LMV; por lo cual se inició el tratamiento con Albendazol antes de tener estudio confirmatorio, con buena respuesta a la segunda dosis con el cese de la fiebre que había mantenido por más de 20 días. Los estudios posteriores confirmaron el diagnóstico de LMV.

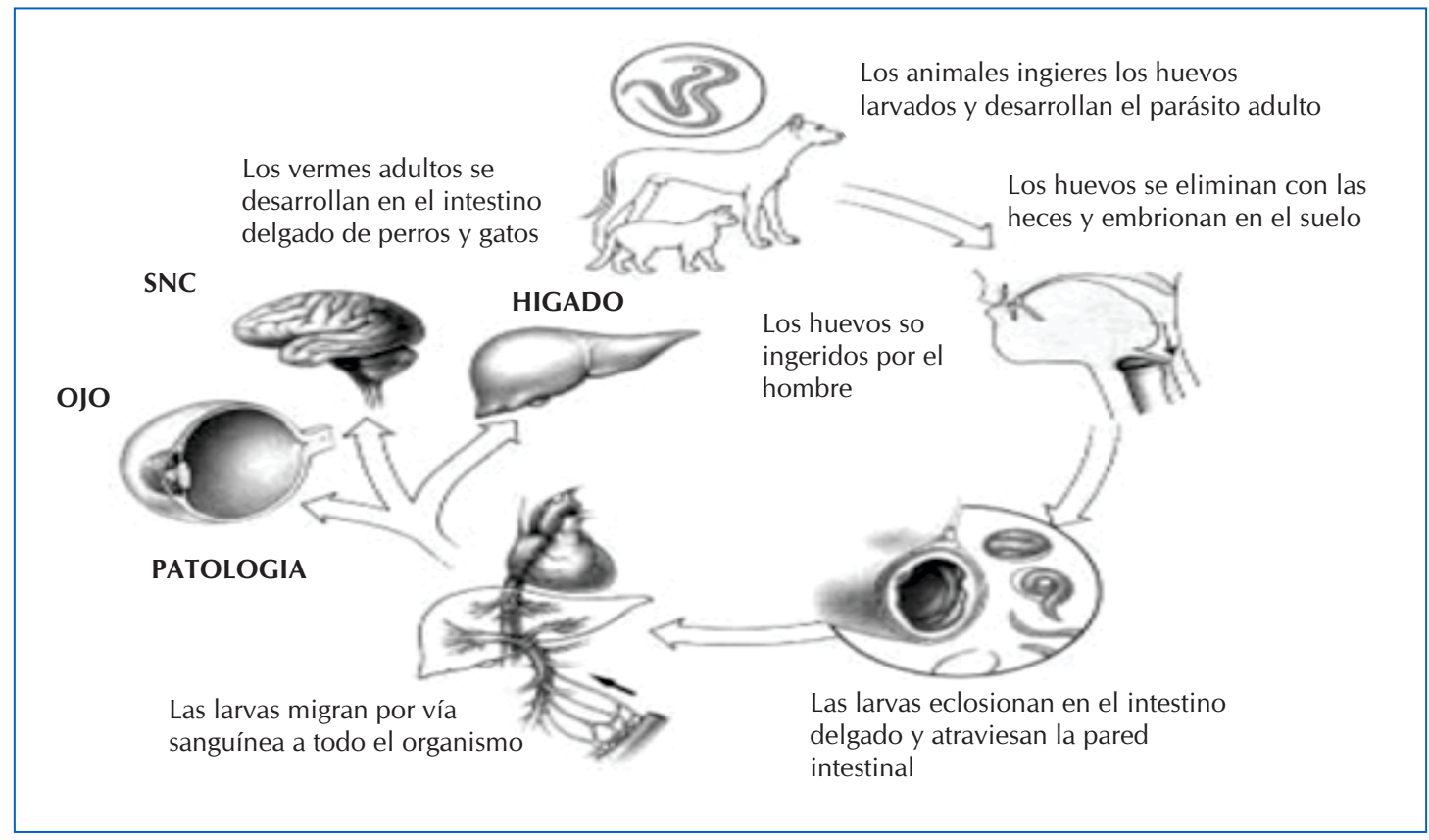

Figura 1. Ciclo de la infección por larvas de toxocarias en la forma de larva migrans visceral.

En la literatura revisada la fiebre no es un síntoma destacable de la LMV y cuando ocurre la misma suele ser de corta duración, aunque se ha descrito un caso de un mes de duración en un adulto de 76 años $^{(43)}$, mientras en el presente caso la misma fue prolongada con una duración de 20 días. La fiebre cedió en forma rápida con la medicación antiparasitaria, lo que hace presumir a la LMV como causante de la misma. La eosinofilia en general persiste por largo tiempo luego del tratamiento, como lo demuestra el presente caso, lo que es muy común en la $\mathrm{LMV}^{(44)}$, normalizándose aún en 
aquellos casos asintomáticos no tratados en un período de 3-5 meses ${ }^{(45)}$. Luego del tratamiento la resolución de los granulomas eosinofílicos del hígado ocurre en una mediana de 4 meses $^{(46)}$. En este paciente, luego de un mes del alta aún persistían los micronódulos hepáticos al igual que la eosinofilia, pero con una tendencia a la disminución progresiva. En el monitoreo de la toxocariasis, cuando se dispone de la ECP, sus valores elevados en sangre constituyen, con la eosinofilia, un marcador importante de actividad de la infección, mientras los valores de la ECP y la IgE sirven para monitoreo del tratamiento de la infección, ya que ambos disminuyen luego de 6 meses $^{(47)}$.

\section{REFERENCIAS}

1. Macpherson CN. The epidemiology and public health importance of toxocariasis: a zoonosis of global importance. Int J Parasitol. 2013;43(12-13):999-1008. doi: http://dx.doi.org/10.1016/j.ijpara.2013.07.004

2. Kroten A, Toczylowski K, Oldak E, Sulik A. Toxocarosis in children: poor hygiene habits and contact with dogs is related to longer treatment. Parasitol Res. 2018;117(5):15131519. doi: http://dx.doi.org/10.1007/s00436-018-5833-7.

3. Chen J, Liu Q, Liu GH, Zheng WB, Hong SJ, Sugiyama H, et al. Toxocariasis: a silent threat with a progressive public health impact. Infect Dis Poverty. 2018;7(1):59. doi: http://dx.doi.org/10.1186/s40249-018-0437-0

4. Woodhall DM, Eberhard ML, Parise ME. Neglected parasitic infections in the United States: toxocariasis. Am J Trop Med Hyg. 2014 May;90(5):810-813. doi: : http://dx.doi.org/10.4269/ajtmh.13-0725

5. Figueiredo SD, Taddei JA, Menezes JJ, Novo NF, Silva EO, Cristóvao HL, et al. Estudo clínico epidemiológico da toxocaríase numa população pediátrica. J Pediatr (Rio J) 2005; 81(2):126-32. doi: https://doi.org/10.1590/S002175572005000300007

6. Martínez M, García H, Figuera L, González V, Lamas F, López K, et al. Seroprevalence and risk factors of toxocariasis in preschool children in Aragua state, Venezuela. Trans R Soc Trop Med Hyg. 2015 Sep;109(9):57988. doi: https://doi.org/10.1093/trstmh/trv059
En el tratamiento de la LMV se han usado el Thiabendazol o el Albendazol, siendo más eficaz el Albendazol a $15 \mathrm{mg} / \mathrm{kg} /$ día, dividida en dos dosis diarias por 5 días, esquema que puede ser repetido según la evolución ${ }^{(48)}$. En las formas oculares LMO al tratamiento con Albendazol se asocia el uso de corticoides orales como la metilpredisona 1 $\mathrm{mg} / \mathrm{kg} /$ día $^{(38,49)}$. Debido al riesgo de la forma ocular que puede desarrollarse en cualquier etapa de la infección, parece justificado implementar tratamiento a todos los niños infectados con toxocara ${ }^{(7,50)}$.

7. Barrios Godoy P, Mauvezin J, Basmadjian Y, Sayagués B, Giachetto G. Toxocariasis: manifestaciones clínicas y de laboratorio en niños asistidos en un prestador integral de salud privado de Montevideo, Uruguay (2014-2018). Rev. Méd. Urug. 2020; 36(1):6-22. doi: http://dx.doi.org/10.2919 3/rmu.36.1.1

8. Altchen J, Nallar M, Conca M, Bancardi M, Feilij H. Toxocariasis: aspectos clínicos y de laboratorio en 54 pacientes. An Pediatr (Barcelona). 2003; 58(5):425-431. doi: http://dx.doi.org/10.1016/S1695-4033(03)78088-6

9. Strube C, Huer L, Janacek E. Toxocara spp. Infections in paratenic hosts. Veterinary Parasitology. 2013;193:375389. doi: https://doi.org/10.1016/j.vetpar.2012.12.033

10. Fan CK, Liao CW, Cheng Y. Factors affecting disease manifestation of toxocariasis in humans: genetic and environment. Vet Parasit. 2013;193(4): 342-353. doi: https://doi.org/10.1016/j.vetpar.2012.12.030

11. Arighi P, Hausbauer G, Vazquez M, Nastri M. Sindrome de larva migrans visceral y absceso hepático. Reporte de un caso. Arch Argent Pediatr 2018; 116(6):753-756

12. Fillaux J, Magnaval JF. Laboratory diagnosis of human toxocariasis. Veter Parasit. 2013; 193(4):327-336. doi: https://doi.org/10.1016/j.vetpar.2012.12.028

13. Sowemimo OA, Lee YL, Asaolu SO, Chuang TW, Akinwale OP, Badejoko BO, et al. Seroepidemiological study and associated risk factors of Toxocara canis 
infection among preschool children in Osun State, Nigeria. Acta Trop. 2017;173:85-89. doi: https://doi.org/1 0.1016/j.actatropica.2017.05.030.

14. Duréault A, Perez Valdes C, Weber L, Ogna A, Sempoux C, Manuel O, Delaloye J. Toxocariasis, a neglected disease in Switzerland?. Rev Med Suisse. 2017;13(558):815-819.

15. Rostami A, Riahi SM, Holland CV, Taghipour A, Khalili-Fomeshi M, et al. Seroprevalence estimates for toxocariasis in people worldwide: A systematic review and meta-analysis. PLoS Negl Trop Dis. 2019;13(12):e0007809. doi:https://doi.org/10.1371/journal.pntd.0007809

16. Espinoza Zaavedra E, Pérez Arellano JL, Sánchez Martín MM, Muro Alvarez A. Parasitosis de interés en nuestro medio: aspectos actuales de la toxocariasis humana. Medicina Integral. 2000;36(10):387-395.

17. Kuenzli E, Neumayr A, Chaney M, Blum J. Toxocariasis-associated cardiac diseases-A systematic review of the literature. Acta Trop. 2016;154:107-20. doi: https://doi.org/10.1016/j.actatropica.2015.11.003

18. Canese A, Domínguez R, Otto C, Ocampos C, Mendonca E. Huevos infectivos de Toxocara, en arenas de plazas y parques de Asunción. Pediatr. (Asunción). 2001;26(2):8-14.

19. Rivarola ME, Vuyk AI, Riveros M, Canese A, Micó GA. Toxocara canis en Población Pediátrica Rural. Pediatr. (Asunción). 2009;36(2):122-126.

20. Sviben M, Cavlek TV, Missoni EM, Galinović GM. Seroprevalence of Toxocara canis infection among asymptomatic children with eosinophilia in Croatia. J Helminthol. 2009;83(4):369-71. doi: https://doi.org/10.1017 /S0022149X09381213

21. Fu CJ, Chuang TW, Lin HS, Wu CH, Liu YC, Langinlur $\mathrm{MK}$, et al. Seroepidemiology of Toxocara canis infection among primary schoolchildren in the capital area of the Republic of the Marshall Islands. BMC Infect Dis. 2014;14:261. doi: https://doi.org/10.1186/1471-2334-14-261

22. Espinoza YA, Huapaya PH, Roldán WH, Jiménez $S$, Arce Z, Lopez E. Clinical and serological evidence of Toxocara infection in school children from Morrope district, Lambayeque, Peru. Rev Inst Med Trop Sao Paulo. 2008;50(2):101-5. doi: https://doi.org/10.1590/s003646652008000200007

23. Triviño X, Bedregal $P$, Torres $M$, Canales $M$, Alvarado C, Hernandez R. Toxocarosis en Chile: Serie clínica en un centro de pediatría ambulatoria. Parasitol. 1999;23(3-4):113117. doi: http://dx.doi.org/10.4067/S0716-07201999000300008
24. Uhlíková M, Hübner J, Kolárová L, Polácková M. Immunological studies on human larval toxocarosis. Cent Eur J Public Health. 1996 Dec;4(4):242-5.

25. Mohamad S, Azmi NC, Noordin R. Development and evaluation of a Sensitive and Specific Assay for Diagnosis of Human Toxocariasis by use of Three Recombinant Antigens (TES-26, TES-30USM, and TES-120). Journ Clin Microbyol. 2009; 47(6): 1712-1717. doi: http://dx.doi.org/1 0.1128/JCM.00001-09

26. Schwartz JT, Fulkerson TP. An approach to evaluation of persistent Hypereosinophilia in Pediatric patients. Front Inmunol. 2018; 9:1944. doi: http://dx.doi.org/10.33 89/fimmu.2018.01944

27. Fischman L, Pavlove M. Sindrome Hipereosinofílico. Hematol. 2007;11(3):220-242.

28. Magnaval JF, Faufingue JH, Morassin B, Fabre R. Eosinophil cationic protein, specific $\operatorname{IgE}$ and $\operatorname{IgG} 4$ in human toxocariasis. J Helminthol. 2006;80(4):417-23. doi: http://dx.doi.org/10.1017/joh2006369. PMID: 17125552.

29. Ciarmela ML, Pezzani BC, Minvielle MC. Toxocariasis, Intestinal Parasitoses and Eosinophilia in Schoolchildren from Argentina. Curr Health Sci J. 2016;42(1):5-11. doi: http://dx.doi.org/10.12865/CHSJ.42.01.01

30. Samudio A, Matto I. Eosinofilias en Pediatría. Experiencia del Departamento de Hemato-Oncología Pediátrica del Hospital de Clínicas Período 2007-2014. Resúmenes de Temas Libres XIV Congreso Paraguayo de Pediatría. Pediatr. (Asunción) 2014; 41(Suplemento 2014):44.

31. Bede O, Szénási Z, Danka J, Gyurkovits K, Nagy D. Toxocariasis associated with chronic cough in childhood: a longitudinal study in Hungary. J Helminthol. 2008;82(4):357-63. doi: http://dx.doi.org/10.1017/S0022149 X0804827X.

32. Li L, Gao W, Yang X, Wu D, Bi H, Zhang S, Huang M, Yao X. Asthma and toxocariasis. Ann Allergy Asthma Immunol. 2014;113(2):187-92. doi: http://dx.doi.org/10.10 16/j.anai.2014.05.016

33. Momen T, Esmaeil N, Reisi M. Seroprevalence of ToxocaraCanis in Asthmatic Children and its Relation to the Severity of Diseases - a Case-Control Study. Med Arch. 2018;72(3):174-177. doi: http://dx.doi.org/10.5455/meda rh.2018.72.174-177

34. Lozano Beltran DF, Suarez Barrientos E, Ortuño E, Cruz Torrico M, Cordova Rojas M, Getaz Jimenez G, et al. Relación entre asma y toxocariasis en pacientes pediátricos en Cochabamba, Bolivia. Gac Med Bol. 2011;34(2):76-79. 
35. Gétaz Schaller L, Samalvides Cuba F, Breña Chavez J, Torrejon D, Maguiña Vargas C. Relación entre Toxocariasis y asma: Estudio prospectivo en niños del Hospital Cayetano Heredia, Lima, Perú. Acta méd. peruana. 2007; 24(2):46-50.

36. Quercia RT, Sgroi I, Fandiño AC, Costa M, Scovenna M, Parra A. Aspectos epidemiológicos, diagnósticos y de tratamiento de la toxocaríasis ocular. Med. Infant. 2015; 22(2):98-105.

37. Zarnowska-Prymek H. Zwiekszenie swoistości laboratoryjnej diagnostyki toksokarozy. Wiad Parazytol. 2001;47(3):489-96.

38. Puerto Sanabria RS, Tovar S. Infección al Sistema Nervioso por toxocara canis en Hospital Escuela Universitario, Honduras. Rev. fac. cienc, méd (Impr.) 2016; 13(2): 47-51.

39. Marx C, Lin J, Masruha MB, Rodríguez MG, Rocha AJ, Villanova LC. Toxocariasis of the CNS symulating acute disseminated encephalomyelitis. Neurolgy. 2007; 69(8): 8067. doi: http://dx.doi.org/10.1212/01.wnl.0000267664.53595.75

40. Moreira-Silva SF, Rodrigues MG, Pimenta JL, Gomes $\mathrm{CP}$, Freire LH, Pereira FEL. Toxocaríase do sistema nervoso central: com descrição de dois casos. Rev. Soc. Bras. Med. Trop. 2004;37(2):169-174. doi: https://doi.org/ 10.1590/S0037-86822004000200011

41. Hellmich T, Martínez G. Hipereosinofilia persistente en pacientes pediátricos. Toxocariasis; un diagnóstico a considerar. Resúmenes de Temas Libres XIV Congreso Paraguayo de Pediatría. Pediatr. (Asunción) 2014; 41(Suplemento 2014):53-57.

42. Sayar D, Masilis A, Kassem E, Kleim A. Toxocariasis as a cause of hypereosinophilia. Harefuah. 2009;148(1):14-16.

43. García Cuervo DA, Miranda Folch JJ, Trimiño Galindo L, Jiménez Álvarez A, Guardarrama Linares L, Suárez Díaz T. Larva migrans visceral. Presentación de un caso. Rev. Med. Electrón. 2018; 40(2):454-462.
44. Ayala J, Chamorro LA. Toxocariasis con hipereosinofilia persistente. A propósito de un caso. Resúmenes de Temas Libres XIV Congreso Paraguayo de Pediatría. Pediatr. (Asunción) 2018;45:81-82.

45. Yoon SY, Baek S, Park SY, Shin B, Kwon HS, Cho YS, et al. Clinical course and treatment outcomes of toxocariasisrelated eosinophilic disorder. Medicine (Baltimore). 2018;97(37):e12361. doi: https://doi.org/10.1097/MD.00000 00000012361

46. Kwon JW, Kim TW, Kim KM, Lee SH, Cho SH, Min KU, et al. Clinical features of clinically diagnosed eosinophilic liver abscesses. Hepatol Int. 2011;5(4):949-54. doi: https://doi.org/10.1007/s12072-011-9272-0

47. Niedworok M, Sordyl B, Borecka A, Gawor J, MałeckaPanas E. Ocena eozynofilii, stezenia immunoglobuliny E i eozynofilowego białka kationowego podczas leczenia toksokarozy. Wiad Parazytol. 2008;54(3):225-30.

48. Stürchler D, Schubarth P, Gualzata M, Gottstein B, Oettli A. Thiabendazole vs. albendazole in treatment of toxocariasis: a clinical trial. Ann Trop Med Parasitol. 1989;83(5):473-8. doi: https://doi.org/10.1080/00034983.1 989.11812374

49. Rossi ML, Argüelles MV, Riera MJ, Magurno M. Toxocaríasis ocular de presentación atípica en adolescente de 18 años. Oftalmol Clin Exp 2010;3(4):155-158.

50. Wiśniewska-Ligier M, Woźniakowska-Gęsicka T, Sobolewska-Dryjańska J, Markiewicz-Jóźwiak A, Wieczorek M. Analysis of the course and treatment of toxocariasis in children-a long-term observation. Parasitol Res. 2012;110(6):2363-71. doi: https://doi.org/10.1007/s0 0436-011-2772-y 